active principles, the heritage of folk medicine, and so on. A list is provided of plants under English common-names -not always correct-with the conditions they are held to alleviate; and under latin names with an illustration and general description that would not be very helpful to a non-botanist, and is followed by a list of complaints and illnesses, with the plants used. This list unfortunately does not always tally with the first and is followed by yet another on healing substances and their effectiveness. This last list is about the parts of the plants used, their constituents, and how they are processed. It is the most informative of the sections, but would be more helpful if sources

\section{Plant breeding for disease resistance}

Plant Breeding for Pest and Disease Resistance. By G. E. Russell. Pp. 485. (Butterworths: London, 1978.) $£ 25$.

DURING the past few years, radical changes have taken place in the strategies used by plant breeders when breeding for resistance to plant pests and diseases. Following the discovery by Biffen in 1907 that resistance to a disease was often conferred by a single gene, known as a major gene, many plant breeders used small nlimbers of major genes to obtain resistance. It was subsequently found, however, that resistance based on a single major gene, or a few such genes, frequently broke down within a few years of its introduction into a crop plant, due to the pathogen producing new races capable of overcoming that gene. G. E. Russell has been actively concerned in devising new breeding strategies designed to provide durable resistance, and this book reflects his experience and methods of approach.

The first part of the book is devoted to a brief but useful summary of methods used for the control of plant diseases, and to a consideration of the general principles and methods of breeding for resistance. The technical terms and concepts used in plant breeding are clearly defined: though some might not agree with all the definitions offered, they are clear and unambiguous. There is a valuable section on methods, many developed by the author and his colleagues, for the introduction of resistance into commercial crops, and for its assessment when introduced.

Parts II to V are devoted to breeding for resistance to specific groups of pathogenic agents, namely the fungi, of both herbal and planting material were given. For example, Asiatic ginseng is listed, but where the authors' expect would-be growers to buy planting material I do not know; so far as I am aware it is only available from Japan. The book would have been far more useful if the information on each plant were all in one place, instead of being scattered throughout. Lists of suppliers-both of the dried herbs and of planting material-would have increased its value enormously. Once again Mrs Grieve wins overall.

Rosemary Angel

Rosemary Angel is Officer in Charge, Museums Division, Royal Botanic Gardens, Kew, UK.

the bacteria, mycoplasmas and viruses, animal pests and parasitic weeds. In each part, a general introduction is followed by a detailed treatment of a few selected cases in each of which substantial progress has been made in the production of resistant cultivars of crop plants. These case histories fulfill two main functions. For the specialist student, they provide a good and usually up to date account of the present position, with adequate references to the literature. For the more general reader, they illustrate very clearly the ways in which the general principles advocated by the author can be applied to obtain durable resistance to almost any type of pest or disease, with modifications in detail rather than in principle to fit any specific case.

In the final part of the book, some general conclusions on the present position and future prospects in the field are set out. The author is hopeful that by the use of major genes, where they are proved to be durable, and by the use of more sophisticated approaches where they are not, it will be possible to control many major pests and pathogens. The casual reader may at first get the impression that it is suggested that breeding is the only satisfactory solution to pest and disease problems, but in the final section, the author is careful to emphasise that in many situations an integrated control programme involving both resistant cultivars, pesticides and biological and cultural control methods may be required.

This book is a very useful addition to the literature of plant breeding and plant pathology. It will be of value both to the student entering this field, and to the more general reader who is seeking an insight into the principles and methods of modern disease and pest resistance breeding.

J. G. Manners

J. G. Manners is Reader in Biology at the University of Southampton, UK.

\section{Applying digital filters}

Digital Filters and their Applications. By V. Cappellini, A. G. Constantinides and P. Emiliani. Pp. 393. (Academic: London, New York and San Francisco, 1978.) £20.50; $\$ 42$.

THE appearance of this volume in a series on Techniques in Physics is something of a landmark, for it is only recently that filters have ceased to be studied almost exclusively by electrical engineers, and written about for readers with formal training in that field. The intensive study of two-dimensional filters is also a recent development, spurred on by the need to clean up satellite pictures and by a variety of biomedical and geological needs. In connection with these and related applications, many scientists with no engineering background have been using - and rediscovering-filters to improve their "signals", and the present authors have therefore attempted to compose a text that would appeal to such an audience as well as to engineers. This is no easy task, for the types of presentation likely to satisfy these two categories of reader are rather different, particularly in the handling of mathematical details and in the need to put the material under discussion in context. The solution adopted by the authors, who present a great deal of material with very little comment or explanatory text to help the reader to assimilate it, is not at all satisfactory. It is certainly possible to look up any number of facts about filters, both FIR and IIR, their software and hardware implementations and noise, but it is extremely difficult to get any feel for their power or suitability. The section entitled "Description of some windows" is an example of this: two formulae tell us how to distinguish a Hamming window from a Hanning window but not why two nearly identical windows should coexist. The formulae for numerous other windows are also given, sometimes with some indications about their origin but not always.

This is a disappointing book, therefore, except as an aide-memoire for finding formulae and definitions. The authors' object was certainly laudable but for the time being the pair of books on digital signal processing by Rabiner and Gold and by Oppenheim and Shafer (Prentice-Hall, 1975) remain the most useful source of information on these matters.

P. W. Hawkes

P. W. Hawkes is Maitre de Recherches at Laboratoire d'Optique Electronique du CNRS, Toulouse, France. 